\title{
Localization and hybridization of the electronic states in thin films of $\mathrm{Ag}$ on $\mathrm{V}(100)$
}

\author{
P. Lazić, Ž. Crljen, and R. Brako* \\ R. Bošković Institute, P.O. Box 180, 10002 Zagreb, Croatia \\ (Received 4 May 2004; revised manuscript received 9 September 2004; published 7 April 2005)
}

\begin{abstract}
We have studied the electronic states in one- to five-layers-thick Ag films on V(100), by means of $a b$ initio density-functional calculations. Due to the mismatch of the electronic structure of $\mathrm{Ag}$ and $\mathrm{V}$, quantum-well states of both $s p$ and $d$ character localized on Ag films are formed. We find that the hybridization of the $\mathrm{Ag}$ quantum-well states with the $\mathrm{V}$ orbitals is nevertheless important, and must be taken into account in order to fully understand the observed properties, in particular the energies and the dispersion of the photoemission peaks in angle-resolved photoemission spectroscopy experiments.
\end{abstract}

DOI: 10.1103/PhysRevB.71.155402

PACS number(s): 73.61.At, 73.21.Fg, 73.20.At, 71.15.Mb

\section{INTRODUCTION}

Ultrathin metallic films grown on a different metal substrate show interesting structural and electronic properties. Some grow in registry with the substrate, while others form a variety of commensurate or incommensurate structures, develop grain boundaries, etc. Of particular interest are the systems which exhibit quantum size effects (QSE), where the electronic states in the films, with thickness typically from one up to several tens of layers, show quantized motion in the direction perpendicular to the surface plane. Quantumwell (QW) states or quantum-well resonances can be formed, depending on the degree of mismatch between the electronic structure of the two metals, and the properties can vary a lot over the extent of the two-dimensional surface Brillouin zone (BZ).

Several experimental techniques can be used to probe the electronic properties of thin metallic films, and in particular the quantum-well states. For an overview see, e.g., a recent review paper. ${ }^{1}$ Most commonly used are angle-resolved photoemission spectroscopy (ARPES) and the scanning tunneling microscopy (STM). ARPES has been used mostly in the normal emission mode, i.e., in the direction perpendicular to the surface, but angularly resolved spectra in off-normal directions make it possible to study the band structure along any direction of the surface Brillouin zone., ${ }^{2,3}$ The photoemission spectroscopy probes a macroscopic surface region, determined by the size of the incoming photon beam, which means it is best suited for systems of uniform thickness. A well known example of this kind are silver films on a $\mathrm{Fe}(100)$ whisker, which can be grown up to 120 macroscopically uniform atomic layers. ${ }^{4}$ STM is a nanoscopically local method, and offers the possibility to study local structures, such as islands, chains, dots, etc. With the scanning tunneling spectroscopy (STS) technique, in which the differential conductance $(d I / d V)$ is measured, one can reliably determine the energy of quantized electronic states in the range of approximately $1 \mathrm{eV}$ below and above the Fermi level. The constant current STM technique can also give important information about the surface electronic structure, in particular by direct imaging of standing waves formed around surface defects in the low bias voltage (and usually low temperature) regime, and taking the Fourier transform. This method is known as Fourier transform scanning tunneling microscopy (FT-STM). ${ }^{5}$

In this paper, we study the properties of thin films of silver on a V(100) surface. The films can be grown pseudomorphically, i.e., in registry with the substrate, up to a thickness of at least ten layers. It has been recently shown that, by using an appropriate deposition and annealing procedure, films of very high quality can be obtained even on a V(100) surface with oxygen-induced $(5 \times 1)$ reconstruction, because silver atoms displace oxygen and lift the reconstruction. ${ }^{6}$ The silver films grow as (111) layers of fcc structure, slightly tetragonally distorted due to a small mismatch of interatomic distances of the two lattices. The electronic properties of this system are very interesting, due to the profound difference of the band structure of the two metals. In bulk vanadium, the $s$ bands extend approximately from 2.5 to $7 \mathrm{eV}$ below the Fermi level, while the $d$ bands exist between the Fermi level down to $3 \mathrm{eV}$. The order is reverse in bulk silver which has $d$ bands between 3.5 and $6 \mathrm{eV}$ below the Fermi level, and the broad $s p$ bands extend from the Fermi level down to $7 \mathrm{eV}$. This symmetry mismatch is particularly important in the center of the two-dimensional Brillouin zone of the $\mathrm{V}-\mathrm{Ag}$ (100) interface, where it amounts to a symmetry gap which prevents hybridization of certain electron bands across the interface. In ultrathin $\mathrm{Ag}$ films on $\mathrm{V}(100)$, it has a consequence that both the $s p$ orbitals of $\mathrm{Ag}$ close to the Fermi level and $d$ orbitals at larger binding energies form QW states which are mostly localized within the Ag layers. In normal ARPES, which selects electronic states at the center of the twodimensional Brillouin zone, the QW states appear as strong narrow peaks. ${ }^{7,8}$

A number of approaches have been used in the past in order to describe the electronic properties, in particular the QW states, in ultrathin metallic films. Quasi-one-dimensional models, such as a square well potential ${ }^{9}$ or the phase accumulation model, ${ }^{10}$ have been successfully used to interpret the energies of QW states. More sophisticated methods have also been used, such as the tight-binding approach ${ }^{11}$ and layer-Korringa-Kohn-Rostoker approach. ${ }^{12}$ In a few systems the QW states have been investigated by self-consistent density-functional calculations. ${ }^{13,14}$ There are strong reasons to use the $a b$ initio methods. First, there are no adjustable parameters, and a wide range of calculated structural and 
electronic properties offer the possibility of a detailed comparison with experiments. Also, quantities such as the expected STM profiles and the amplitudes of the wave functions of the QW states, which cannot be obtained in simple approaches, can be calculated.

In a recent paper, we have reported our ab initio calculations of the structure of the $\mathrm{Ag} / \mathrm{V}(100)$ system. ${ }^{6}$ We used the density-functional theory (DFT) approach and calculated the structure and the total energy of silver films on the V(100) surface from one to five layers thick. The calculated interlayer distances, step heights, work functions, and other quantities were in excellent agreement with the experimental findings. In this work, we use the same method to calculate the electronic properties of the $\mathrm{Ag} / \mathrm{V}(100)$ system and compare the results with photoemission experiments.

\section{EXPERIMENTAL STUDIES OF Ag/V(100) FILMS}

STM experiments give important information on structural properties of $\mathrm{Ag} / \mathrm{V}(100)$ films, e.g., the disappearance of the oxygen-assisted $(1 \times 5)$ reconstruction of the bare $\mathrm{V}$ surface upon silver adsorption and annealing, the step heights between $\mathrm{Ag}$ terraces of different number of layers, etc. In STM images, the Ag terraces appear smooth, with small corrugation of square symmetry corresponding to the atomic structure. In a recent experiment, standing waves around impurities ${ }^{15-17}$ have been observed, which indicates that the motion of electrons with energies around the Fermi level is largely two-dimensional, confined to the Ag films. This is one of the rare examples where the oscillatory wave patterns at metal surfaces have been observed at room temperature.

In photoemission spectra along the surface normal, the quantum-well states of predominantly $s p$ character appear as strong well-defined peaks in the energy region between the Fermi level and $2.5 \mathrm{eV}$ below it. They are narrow and the position of the maximum does not depend on the energy of the incident light, which means that they are localized on the silver films. The peak position is a characteristic of the number of $\mathrm{Ag}$ layers, and the appearance of one or more peaks in the photoemission spectra can be used to determine the thickness and the degree of perfection of the films. Offnormal photoemission measurements make it possible to determine the dispersion relation of the QW states. ${ }^{18}$ Away from the center of the surface Brillouin zone, i.e., at finite $k_{\|}$, the photoemission peaks of the QW states broaden and the dependence upon $k_{\|}$often deviates from the parabolic freeelectron behavior. Several of the theoretical approaches mentioned in the Introduction have been used to treat the $s p \mathrm{QW}$ states of $\mathrm{Ag} / \mathrm{V}(100)$ films, including a simple square potential, ${ }^{19}$ the phase accumulation model, ${ }^{19}$ the layerKorringa-Kohn-Rostoker approach, ${ }^{12}$ and the tight-binding calculations. ${ }^{8}$ These calculations have made it possible to assign the experimentally observed QW states to different families according to the number of nodes of the wave function, for up to eight layers or so. ${ }^{12}$

The energy region from 2.5 to $7 \mathrm{eV}$ below the Fermi level in photoemission is dominated by features derived from $d$ bands of $\mathrm{Ag}$. A number of sharp peaks are observed in normal photoemission, implying that some $d$ orbitals form QW states localized within silver films.

\section{CAlCUlations}

The density-functional calculations were performed using the DACAPO program with ultrasoft pseudopotentials for the Perdew-Wang exchange-correlation functional, in the generalized gradient approximation (GGA). The computational procedure has been described in considerable details in Ref. 6 , and here we only give a brief overview. Seven layers of vanadium were used to describe the bcc V(100) substrate, on which one to five silver layers were added. Since periodic boundary conditions were used in all three spatial directions, a layer of vacuum of around $15 \AA$ was added on top of silver. The structure was allowed to fully relax in the direction perpendicular to the surface, except for the three bottom V layers, which were kept fixed at the bulk interatomic distance. We used a mesh of $12 \times 12 k$ points in the directions along the surface plane. Once the full self-consistency of the relaxed structure was achieved, we made additional calculations with many more $k$ points (typically 36 from the center to the edge of the BZ) along the two high-symmetry directions of the surface Brillouin zone, in order to obtain the dispersion relations and the wave functions of the KohnSham eigenstates in more detail. Since in these calculations the $k$ points do not cover uniformly the whole twodimensional Brillouin zone, the potential determined beforehand in self-consistent calculations is used.

We use the calculated electronic eigenstate energies and wave functions for comparison with experiments, in particular photoemission. However, the Kohn-Sham densityfunctional approach is designed primarily for an accurate calculation of the total energy of the system. By varying the positions of the atoms, one can determine the equilibrium atomic configuration, the interatomic potential around the equilibrium, etc. The electronic eigenstates are obtained as an intermediate result and their quality and suitability for the description of various electronic processes is not guaranteed. The method is particularly unreliable for empty electronic states above the Fermi level, which do not contribute towards the total energy of the system and are therefore not included in the energy functional. In fact, it is possible to perform the DFT calculations using just enough orbitals to accommodate all the electrons, at the expense of the rate of convergence, in which case the empty states will be obviously inadequate. Even if sufficiently many orbitals are included, the vacuum layer separating the films in the direction perpendicular to the surface may not be large enough to prevent the coupling of states above the Fermi level due to a finite probability of tunneling through the vacuum. Care should be exerted if empty states are used, e.g., in the analysis of STM images. Nevertheless, it has repeatedly been found that, if adequate precaution is used, the calculated electronic eigenstates yield a good description of the electronic structure of the solid. From comparison with other calculations, we have concluded that our calculation indeed gives good electronic structure, apart from some $d$ bands of silver being too close to the Fermi level, which will be dis- 


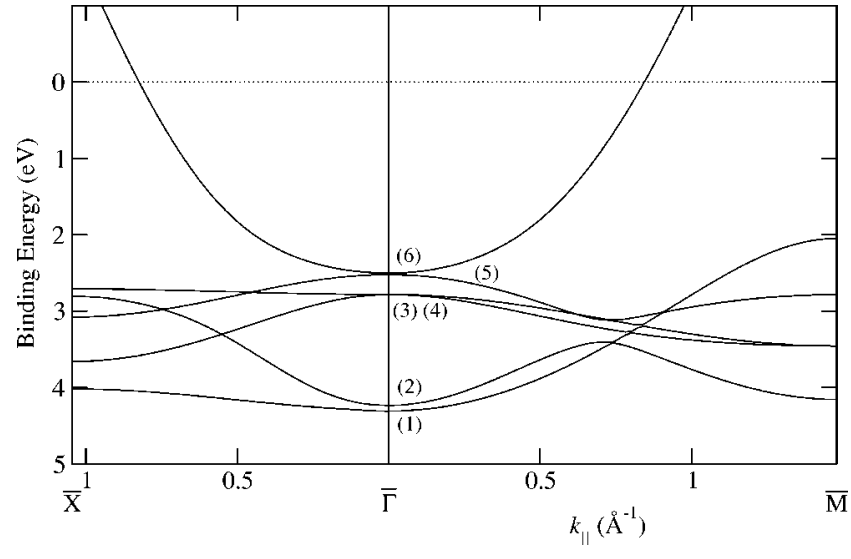

FIG. 1. The energies of electronic states of an unsupported Ag monolayer of square symmetry, with lattice constant corresponding to interatomic distance on a $\mathrm{V}(100)$ surface. The energies are calculated using DFT, in the high-symmetry directions of the surface Brillouin zone. In the center of the Brillouin zone, the character of the states is, starting from the lowest energy, (1) $d_{x^{2}-y^{2}}$, (2) $d_{z z}$ with some $s$ admixture, (3),(4) degenerate $d_{x z}$ and $d_{y z}$, (5) $d_{x y}$, and (6) predominantly $s$.

cussed in detail later on. Apparently, the $d$ bands have been a problem in a number of DFT calculations of the electronic structure of noble metals.

\section{RESULTS AND COMPARISON WITH EXPERIMENT}

\section{A. The Kohn-Sham eigenstates}

We first made density-functional calculations of an unsupported Ag monolayer (i.e., a layer of silver atoms surrounded on both sides by vacuum) of the same geometry as Ag atoms in a $1 \mathrm{ML}$ film adsorbed on $\mathrm{V}(100)$. The energies of the Kohn-Sham eigenstates are shown in Fig. 1, along the highsymmetry directions of the surface Brillouin zone $\bar{\Gamma}-\bar{X}$ and $\bar{\Gamma}-\bar{M}$. The states between 2.5 and $4.5 \mathrm{eV}$ binding energy with relatively flat dispersion curves are $d$ bands, while the $s$ band shows a free-electron-like dispersion and crosses the Fermi level at around $k_{\|}=0.8 \AA^{-1}$ in both calculated directions. Around the center of the Brillouin zone the $s$ orbitals also contribute some weight to the $d_{z z}$ band, second from below, which is possible as both belong to the same completely symmetric representation of the symmetry group at $\bar{\Gamma}$ point.

Next we calculated the Kohn-Sham energy bands of bulk vanadium and projected them onto the directions relevant for the surface calculations. In Fig. 2(a), we show the bands along the $\Gamma-\Delta-\mathrm{H}$ direction, i.e., the $k_{z}$ direction in our surface calculations, which projects onto the $\bar{\Gamma}$ point of the (100) vanadium surface Brillouin zone. In Fig. 2(b), we show the bands in the high-symmetry directions of the surface Brillouin zone, for 20 values of $k_{z}$. There are no true energy gaps at or near the $\bar{\Gamma}$ point. However, according to Fig. 2(a) in the energy region between $2.3 \mathrm{eV}$ and the Fermi level, only states of $\Delta_{2}$ symmetry exist. Consequently, the electronic states of silver adlayers at $\bar{\Gamma}$ which are totally rotationally symmetric with respect to the $z$ axis (i.e., $s$ and $d_{z z}$ ) which fall in this energy range cannot hybridize with the substrate bands. This is the so-called symmetry gap which allows the existence of quantum-well states in adsorbate layers even when the substrate has no true energy gaps. Of course, the symmetry incompatibility ceases to be exactly true as soon as we move away from the $\bar{\Gamma}$ point. Thus we expect that the photoemission peaks from such adsorbate QW become broader when measured away from the normal direction, i.e., for a finite $k_{\|}$. This is unlike the QW states on substrates with a true energy gap, where narrow photoemission peaks can be observed for large $k_{\|}$, until they hit either the Fermi level or the edge of a projected band.

At binding energies larger than approximately $3 \mathrm{eV}$, the converse is true. According to Fig. 2, only electronic states of the $\Delta_{1}$ symmetry exist at the $\bar{\Gamma}$ point, which implies that there is a symmetry gap for $d$ states other than $d_{z z}$ and the respective QW states may occur.
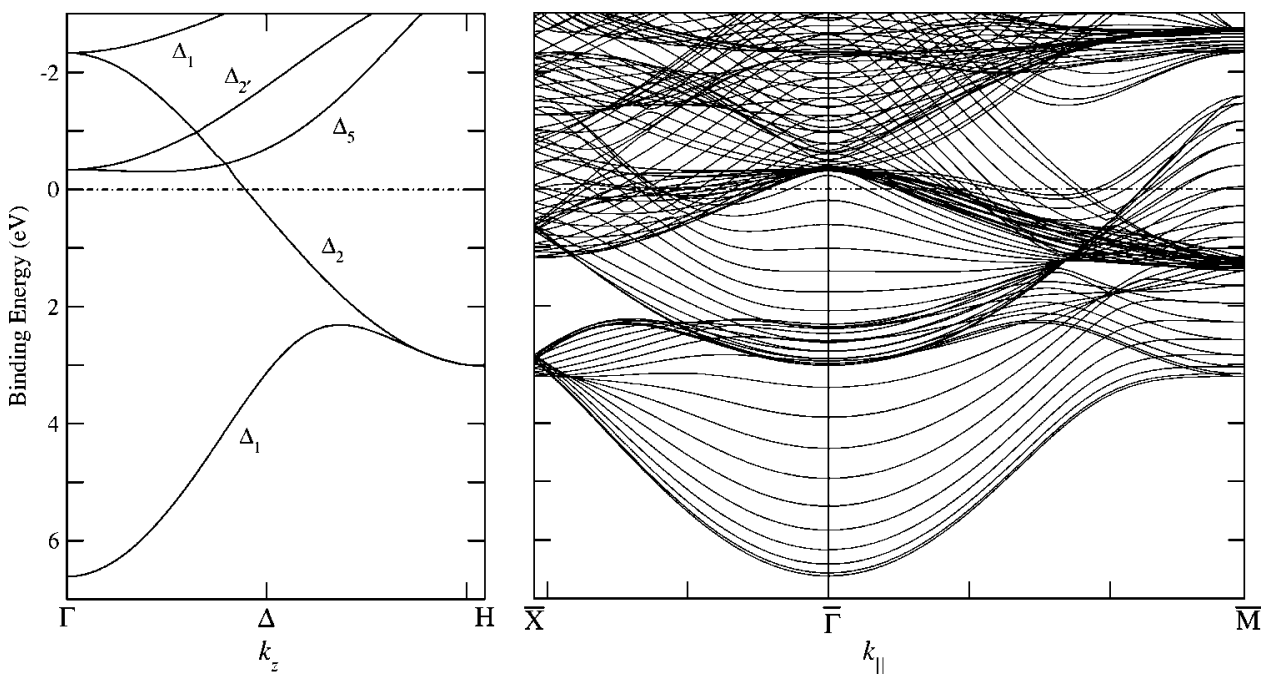

FIG. 2. The electronic structure of bulk vanadium. (a) In the direction normal to the V(100) surface. The bands are labeled by their group representation. (b) Projection along high-symmetry directions of the (100) surface Brillouin zone, for 20 values of $k_{z}$. 


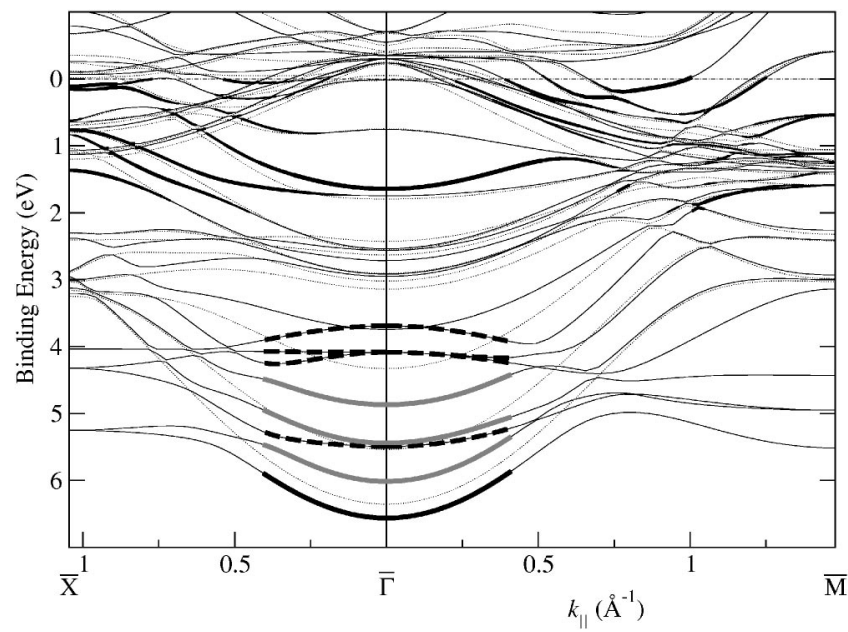

FIG. 3. Similar as Fig. 1, but for a monolayer of Ag on seven layers of vanadium (100) (full lines) and seven layers of V only (dotted lines). Around the center of the Brillouin zone, we have denoted by thicker lines the states of the $\mathrm{Ag} / \mathrm{V}(100)$ system which have a large amplitude on the silver layer. Black lines: states with $s$ character, gray lines: Ag states of $d_{z z}$ symmetry, which hybridize strongly with the $s$ band of vanadium, dashed lines: Ag states of other $d$ symmetries. In the region between $1.65 \mathrm{eV}$ binding energy and the Fermi level, we have calculated the magnitude of various states on the silver layer, and represented it by lines of different thickness. For a detailed discussion, see the text.

Finally, we come to the results for the combined vanadium-silver systems. The dispersion relations of the eigenstates for $1 \mathrm{ML}$ and $2 \mathrm{ML} \mathrm{Ag}$ films on seven layers of $\mathrm{V}(100)$, calculated as explained in Sec. III, are shown in Figs. 3 and 4. The thin dotted lines in Fig. 3 are the results for bare vanadium substrate consisting of seven layers, which are similar to the bulk dispersion relations projected onto the surface, Fig. 2(b), but more coarsely spaced because of the small number of layers. The full lines are the results of the calculation with the silver adlayer. It is easy to recognize

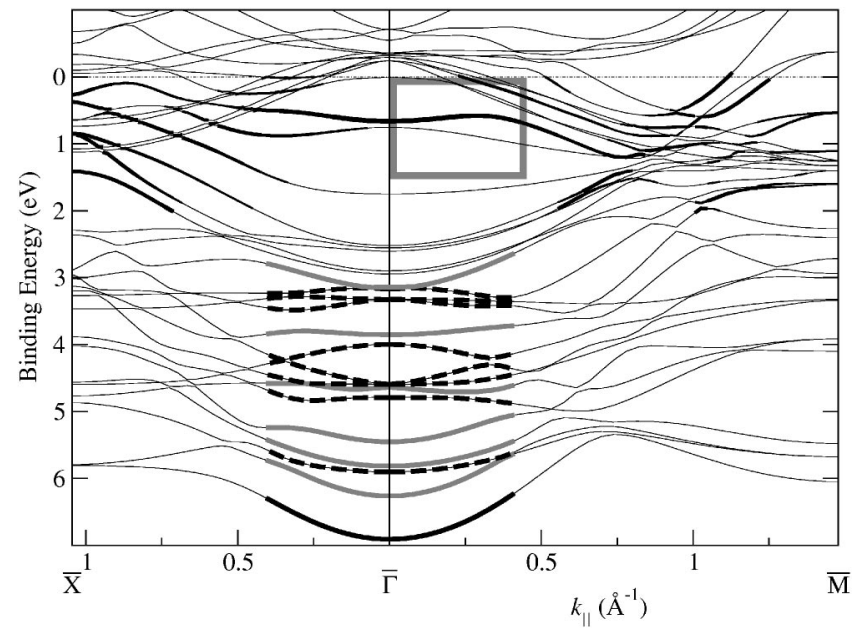

FIG. 4. Similar to Fig. 3, but for two layers of $\mathrm{Ag}$ on seven layers of vanadium. The gray box denotes the $E-k$ region, which has been investigated experimentally by off-normal ARPES in Refs. 15 and 16. the electronic states which are localized mostly in vanadium, as they run very close to the dotted lines of the clean $\mathrm{V}$, while the states associated with silver do not have a corresponding dotted line. The states which at $k_{\|}=0$ have a large weight on silver atoms are shown by thick lines around the center of the Brillouin zone. The different line styles denote states of different symmetry, which has been deduced from the density of states projected onto atomic orbitals. In the energy range between the Fermi level and $2 \mathrm{eV}$, we have gone a step further, and calculated the integral over the silver atoms of the density of various eigenstates, for the full range of $k_{\|}$ vectors. The results are shown as lines of various thickness, the thick segments corresponding to states localized at silver atoms by more than around 35\%. The reasoning behind this approach is that the calculated integrals are roughly proportional to the intensity of the photoemission peaks in ARPES experiments, since photoelectrons originating from deeper layers have a small probability of leaving the solid without being scattered. The most prominent among the thick lines are the quantum-well states with energy of $1.65 \mathrm{eV}$ for $1 \mathrm{ML}$ and $0.66 \mathrm{eV}$ for $2 \mathrm{ML}$ films at the $\bar{\Gamma}$ point. They appear as strong narrow peaks in ARPES experiments, as discussed in the following subsection.

Comparing the states of $1 \mathrm{ML} \mathrm{Ag} \mathrm{films} \mathrm{on} \mathrm{vanadium,} \mathrm{Fig.}$ 3 , with the states of the unsupported Ag monolayer, Fig. 1, it is evident that the $\operatorname{Ag} d$ bands (except $d_{z z}$ ), drawn by dashed lines, are pulled down to lower energies, while the shape of the dispersion curves remains quite similar. This can be attributed to the fact that the effective potential well confining the states within the $\mathrm{Ag}$ film is wider in the $\mathrm{Ag} / \mathrm{V}(100)$ case than in the (rather unrealistic) self-standing Ag film, but otherwise the $d$ states do not hybridize significantly with the vanadium substrate. The situation is very different in the case of $s p$ and $d_{z z}$ derived bands, which fall largely within the energy range of the vanadium $s$ band and hybridize strongly with it. Around the center of the surface Brillouin zone, the weight of the $\mathrm{Ag} s p$ orbitals is transferred mostly to the quantum-well state at binding energy $1.65 \mathrm{eV}$ mentioned in the preceding paragraph, and to the state at $6.56 \mathrm{eV}$, just below the lower edge of the vanadium $s$ band. The $d_{z z}$ state (with some contribution of Ag $s p$ orbitals) hybridizes strongly with the vanadium $s$ band and contributes to several states, shown as thick gray lines. Their number would increase if we used more than seven layers of $\mathrm{V}$, while their weight on Ag atoms would decrease. Finally, in the limit of a thick vanadium substrate, a broad $\mathrm{Ag} d_{z z}-\mathrm{V} s$ resonance would appear.

A qualitative interpretation of the band structure of $2 \mathrm{ML}$ Ag films, Fig. 4, compared to the $1 \mathrm{ML}$ results is that each $d$ state localized on silver splits into two. The $s p$-derived quantum-well state with highest energy, well localized on $\mathrm{Ag}$, is now at $0.66 \mathrm{eV}$ below the Fermi level.

\section{B. Quantum-well states and resonances}

In order to investigate in detail the character of quantumwell states and resonances, we have made intensity plots of the amplitudes of the wave functions of some Kohn-Sham eigenstates. The wave functions shown in Fig. 5 are for 2 


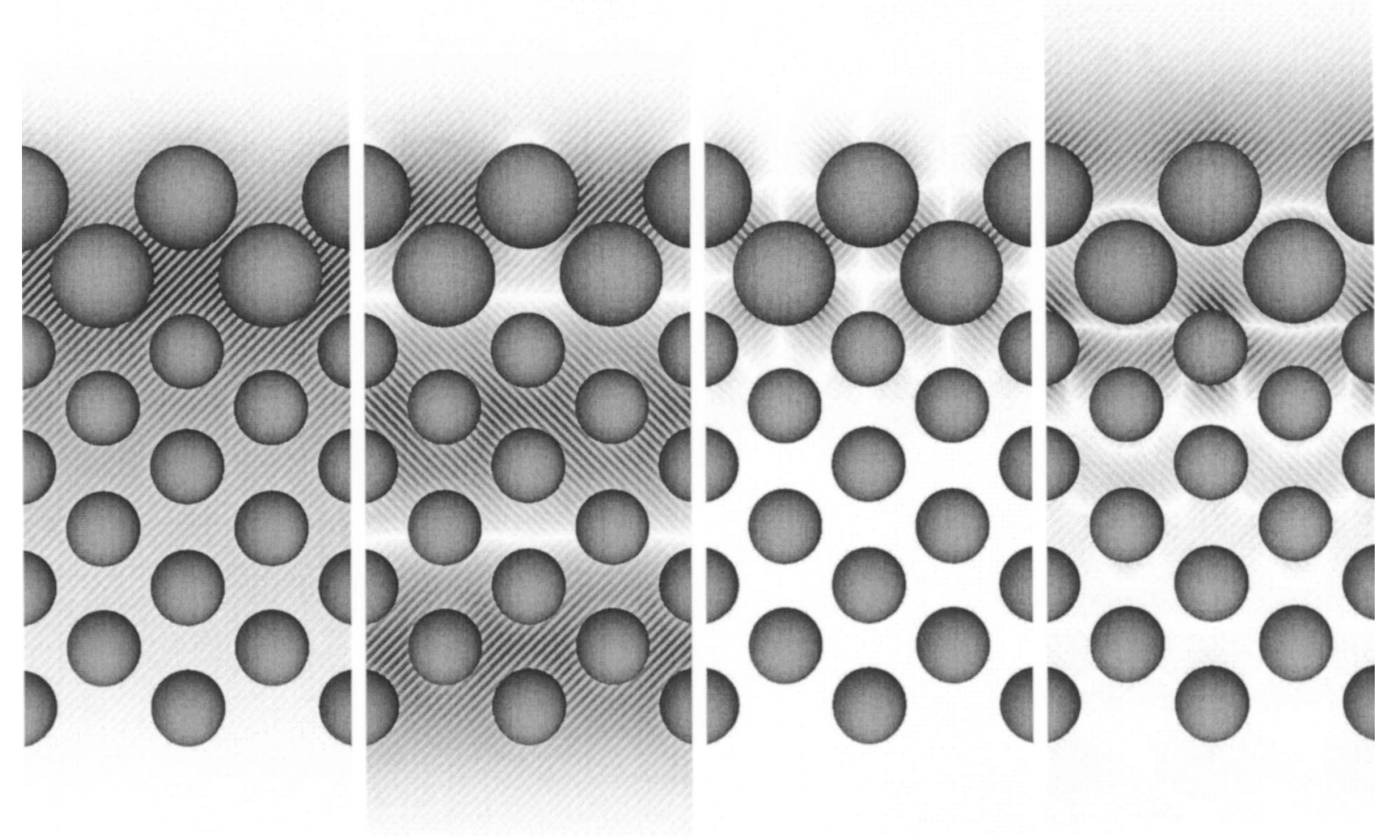

(a)

(b)

(c)

(d)

FIG. 5. The wave functions of some electronic states in films of 2 ML Ag on seven layers of vanadium at the $\bar{\Gamma}$ point, in the plane along $x=y$ and perpendicular to the surface. The solid spheres hide the atomic cores, and the atomic pseudopotentials are defined at distances which lie inside the spheres. The different shadings denote regions in which the wave functions have positive and negative values, respectively. (a) Ag $s p-\mathrm{V} s$ QW state at $6.91 \mathrm{eV}$ binding energy; (b) Ag $s p$ with some $\mathrm{Ag} d_{z z}$, hybridized with V $s$, at $5.45 \mathrm{eV}$; (c) $\mathrm{Ag} d_{x z} \mathrm{QW}$ state at $4.59 \mathrm{eV}$; (d) $\mathrm{Ag} s p-\mathrm{V} d_{z z} \mathrm{QW}$ state at $0.66 \mathrm{eV}$.

ML Ag films on V, but the results for $1 \mathrm{ML} \mathrm{Ag} \mathrm{films} \mathrm{are}$ similar, although with fewer nodes along the $z$ direction. We plotted the wave functions at the center of the surface Brillouin zone $\bar{\Gamma}$ where they can be expressed as a real function by factoring out a constant complex phase. The wave function of the $s p$ QW state at $k_{\|}=0, E=0.66 \mathrm{eV}$, is particularly interesting. It is almost translationally invariant in the directions of the surface plane, with one node, as predicted by the classification of states in the simple potential well models, e.g., as shown in Fig. 10(d) in Ref. 1. However, it has an unexpectedly large amplitude on the first one or two vanadium layers, which is contributed by orbitals of $d_{z z}$ symmetry. In fact, the (approximate) projection of the density of states onto atomic orbitals of various symmetry shows that the wave function consists of approximately half Ag sp and half $\mathrm{V} d_{z z}$ orbitals. Although large on the first $\mathrm{V}$ layer, the contribution of $\mathrm{V}$ orbitals decreases quickly and vanishes within a couple of $\mathrm{V}$ layers, since these orbitals are pulled out from their "natural" energies (i.e., the respective vanadium $d$ bands) and do not propagate into the vanadium bulk.

In Fig. 6, we show the square of the wave function of the $2 \mathrm{ML} \mathrm{Ag} \mathrm{QW} \mathrm{state,} \mathrm{for} \mathrm{three} \mathrm{values} \mathrm{of} \mathrm{the} \mathrm{wave} \mathrm{vector} k_{\|}$ along the $\bar{\Gamma}-\bar{M}$ direction. The functions are shown along the $z$ coordinate at a particular $x_{0}$ and $y_{0}$, chosen in such a way that the line does not intersect any pseudopotential sphere used in our calculation. This was done to avoid the strong variation of the wave functions close to the atomic cores, but apart from that the choice of $x_{0}$ and $y_{0}$ is not significant, since the amplitudes of the wave functions are almost trans-

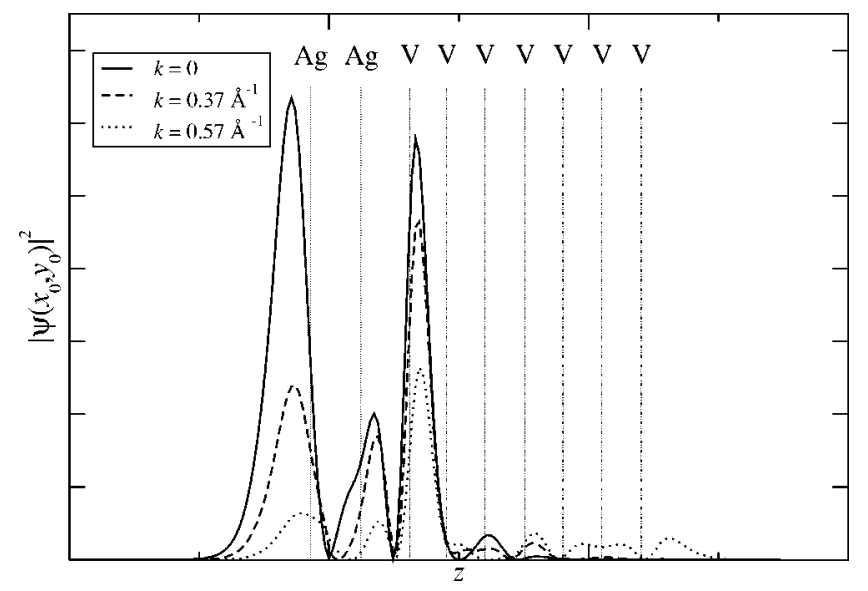

FIG. 6. The square of the wave function of the $2 \mathrm{ML} \mathrm{Ag} s p$ quantum-well state as a function of the coordinate $z$ perpendicular to the surface, for three values of the wave vector $k_{\|}$. The coordinates $x_{0}=0, y_{0}=0.5 a_{\mathrm{V}}$ are chosen so that the line does not intersect any pseudopotential sphere used in the calculation. 


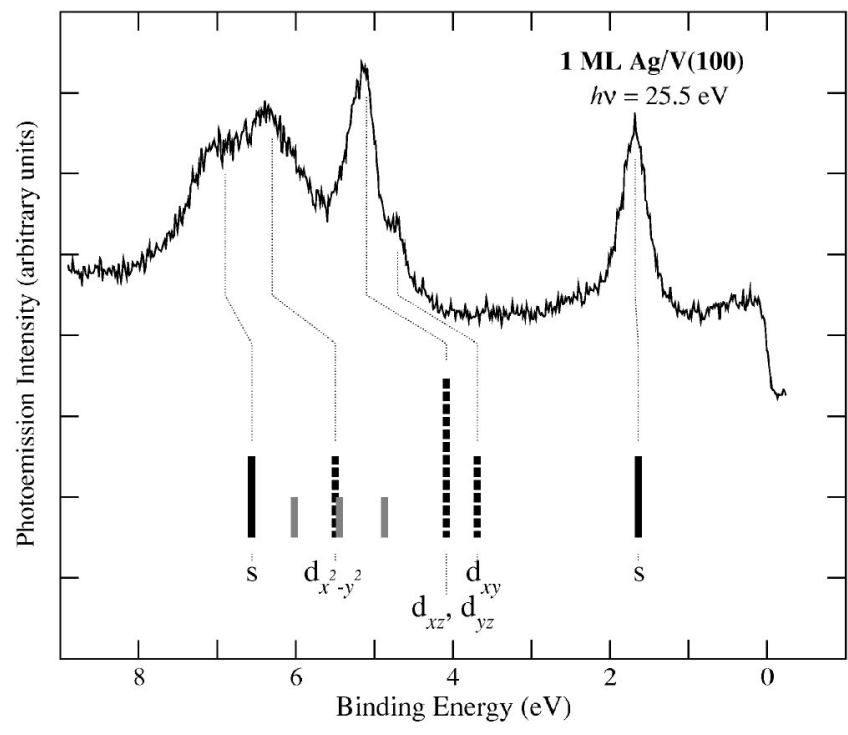

FIG. 7. Experimental normal emission photoelectron spectra of 1 ML Ag films deposited on V(100) surface, and the energy eigenvalues of Ag quantum-well states and resonances obtained in DFT calculations of $1 \mathrm{ML} \mathrm{Ag}$ on a seven layer V(100) substrate. Black bars: the QW states originating from $\mathrm{Ag} s$ orbitals, strongly hybridized with $d_{z z}$ orbitals on the underlying $\mathrm{V}$ atoms (the QW state at $E=1.65 \mathrm{eV}$ ), and with the corresponding $s$ orbitals (the QW state around $E=6.56 \mathrm{eV}$ ). Dashed bars: the $\mathrm{Ag} d \mathrm{QW}$ states of various symmetries. Gray bars: the Ag $s$ QW resonances, which lie within the vanadium $s$ bands. See the discussion in the text.

lationally invariant, as can be seen in the density plot of the QW state at $k_{\|}=0$ in Fig. 5(d). Figure 6 confirms the quantum-well state character of the wave function at $\bar{\Gamma}$, i.e., $k_{\|}=0$, where the symmetry gap is fully effective. At $k_{\|}=0.37 \AA^{-1}$, which is just inside the gray box in Fig. 4, the wave function has a much decreased density outside the first $\mathrm{Ag}$ atom, and extends up to the third and fourth $\mathrm{V}$ layer. Finally, at $k_{\|}=0.57 \AA^{-1}$ the wave function is located mostly on the first two $\mathrm{V}$ layers, but extends through the rest of the slab and has a tail into the vacuum on the other side of our $\mathrm{Ag} / \mathrm{V}$ structure. Thus the state has lost its QW character, and can be more appropriately described as an interface resonance.

These results show that although simple well potential or phase accumulation models may successfully reproduce the energies of the QW states at the $\bar{\Gamma}$ point with an appropriate choice of parameters, only $a b$ initio calculations can give accurate information about the degree of hybridization with $\mathrm{V}$ orbitals, in particular away from the center of the surface Brillouin zone, which can be essential for photoemission cross sections and other properties.

\section{Comparison with experiments}

In Fig. 7, we have plotted the experimental spectra of normal photoemission from a monolayer of $\mathrm{Ag}$ on $\mathrm{V}(100)$ (Ref. 20) and calculated energies at the $\bar{\Gamma}$ point of electronic states localized on the silver adlayer. The experimental spectrum was measured at the ELETTRA synchrotron in Trieste.
The sample was at room temperature, and the energy resolution of the measurement was around $30-50 \mathrm{meV} .{ }^{8}$ The prominent peak at $1.65 \mathrm{eV}$ binding energy has been assigned to the Ag $s p$ quantum-well state, and the one around $5.2 \mathrm{eV}$ to the $\mathrm{Ag} d \mathrm{QW}$ state. The full width at half maximum of both peaks is close to $400 \mathrm{meV}$.

In Ref. 9, a careful analysis of spectra taken in a similar experiment, in which the energy resolution was around $10 \mathrm{meV}$ and the temperature could be varied, showed that at $60 \mathrm{~K}$ the $2 \mathrm{ML} s p$ QW peak width is around $360 \mathrm{meV}$, which was decomposed into various contributions. Based on those results, we can attribute around $200 \mathrm{meV}$ of the linewidth in Fig. 7 to the finite lifetime of the hole (this contribution depends roughly quadratically upon the binding energy of the QW state), $150 \mathrm{meV}$ to impurity and phonon scattering, and the remaining $30-50 \mathrm{meV}$ to the experimental resolution. In Ref. 8, it was found that a large part of the apparent width of the $d \mathrm{QW}$ peak around $5.2 \mathrm{eV}$ comes from the fact that it is a doublet, with the two components split by around $120 \mathrm{meV}$. The splitting is due to the spin-orbit coupling, which is not included in our calculations. Another peak which can be attributed to a $d$ QW state occurs at $4.7 \mathrm{eV}$ binding energy. It is quite weak in the spectrum shown here but has a larger intensity at other photon energies. It is a singlet, with a width around $95 \mathrm{meV} .{ }^{8}$

The results of our calculations are shown as lines of various style and height. The height indicates the degree of localization of the electronic state on the silver adlayer, which gives a rough estimate of the expected photoemission strength. Thus the state of $s$ symmetry at $1.65 \mathrm{eV}$ is shown with a full height line, since it lies in the symmetry gap of vanadium and cannot propagate into the bulk, although it has a strong component on the first $\mathrm{V}$ layer, as already discussed for the $2 \mathrm{ML}$ QW state. Similarly, the $\mathrm{Ag} d$ states of $d_{x y}$ and $d_{x^{2}-y^{2}}$ symmetry at larger binding energies also form QW states due to symmetry mismatch with substrate bands, as do the $d_{x z}, d_{y z}$ states which are shown with a line of double height since they are degenerate at $k=0$. The states derived from $\operatorname{Ag} d_{z z}$ orbitals, however, are shown as half-height gray lines, since they readily hybridize with the $s$ band of the vanadium substrate. These states have also a small admixture of $\mathrm{Ag} s$ orbitals. Finally, we have denoted the state at $6.56 \mathrm{eV}$ also by a black bar, i.e., as a QW state of $s$ symmetry, because in our calculation the wave function decreases approximately exponentially on the deeper vanadium layers. However, our calculation with only seven layers of vanadium underestimates the width of the $s$ band of $\mathrm{V}$, and a more appropriate description of this state may be that of an $s$ resonance at the bottom of the vanadium $s$ band.

The calculated energy of the $s$ QW state at $E=1.65 \mathrm{eV}$ agrees well with the peak in the experimental spectrum. The energies of $d$ quantum-well states, however, are around $1 \mathrm{eV}$ too high compared to the experimental peaks. There is no reason for any shift of the experimental photoemission peaks of the $d$ states compared to $s p$, and this discrepancy appears to be a deficiency of the density-functional calculation. It is indeed known that local density-functional calculations tend to place the $d$ bands of silver (and other noble metals as well) too close to the Fermi energy, as discussed in Ref. 21 and references therein. 
TABLE I. Comparison of calculated and measured energies (in $\mathrm{eV}$, with respect to the Fermi level) of $s p$ quantum-well states of 1-5 ML films of silver on vanadium.

\begin{tabular}{lcc}
\hline \hline $\begin{array}{l}\text { Number } \\
\text { of Ag layers }\end{array}$ & $\begin{array}{c}\text { Experiment } \\
\text { (Ref. 9) }\end{array}$ & Calculation \\
\hline 1 & 1.65 & 1.65 \\
2 & 0.58 & 0.66 \\
3 & & 2.22 \\
4 & 1.43 & 1.61 \\
5 & 0.82 & 0.99 \\
\hline
\end{tabular}

We obtain good agreement for the energies of the $s p$ QW states at the $\bar{\Gamma}$ point for thicker Ag films as well. In Table I, we show the calculated energies of the highest state of $s p$ symmetry, for films from 1 to $5 \mathrm{ML}$ of $\mathrm{Ag}$. In all cases, except possibly for $3 \mathrm{ML}$, the energies fall in the symmetry gap discussed earlier. The results are compared with the values measured in normal photoemission..$^{9}$ The agreement is very good, considering the finite width and the temperature dependence of the experimental peaks. There is no direct measurement of the QW state in $3 \mathrm{ML} \mathrm{Ag} \mathrm{films,} \mathrm{which} \mathrm{are}$ probably structurally unstable. ${ }^{6}$ However, in Ref. 19 photoemission spectra have been reported from Ag films on $\mathrm{V}(100)$ of nominal coverage of $2.5 \mathrm{ML}$, which presumably consist of regions of $2 \mathrm{ML}$ and $3 \mathrm{ML}$ coverage. In Fig. 4 of that reference, the broad peak around $2.3 \mathrm{eV}$, which is the upper edge of the vanadium $s$ band, is assigned to photoemission from a QW state or resonance associated with the 3 ML regions, in good agreement with our calculated value.

The dispersion of Ag QW states can be compared with off-normal ARPES experiments. In Fig. 4, we have marked by a gray box the $E-k_{\|}$region in the $\bar{\Gamma}-\bar{M}$ direction in which the angle-resolved PES spectra of $2 \mathrm{ML} \mathrm{Ag} \mathrm{films} \mathrm{on} \mathrm{vana-}$ dium have been reported. ${ }^{15,16}$ Comparing the thick lines in our calculation to the density plot of the off-normal photoemission intensity in Fig. 6.14 of Ref. 15 and Fig. 2 of Ref. 16 , we see that the agreement is excellent. Both the quadratic dispersion of the QW state around the $\bar{\Gamma}$ point and the subsequent bending down, as well as the transfer of photoemission intensity to the vanadium-induced states which cross the Fermi level at finite $k_{\|}$, are seen in the experimental spectra. It is interesting that our calculations find a quite different behavior in the $\bar{\Gamma}-\bar{X}$ direction, where a state with binding energy slightly larger than the QW state acquires a significant weight on the surface atoms soon after leaving the $\bar{\Gamma}$ point of the Brillouin zone. This direction, however, has not been investigated by ARPES experiments.

In Refs. 15-17, standing-wave patterns around surface defects have been observed in STM images taken on 1-5-ML-thick Ag films on V(100) at room temperature. The defects were mostly point scatterers, and in some cases also surface steps. Such patterns are known to originate from the interference of electrons with predominantly twodimensional character, usually surface states, ${ }^{22}$ on localized potentials. This is a rare example of standing waves in a room-temperature experiment, as most other observations have been done at low temperatures. It indicates a strongly enhanced two-dimensionality of electronic states in silver overlayers with energies around the Fermi level at large values of the wave vector $k_{\|}$. In the unsupported silver monolayer, Fig. 1, the Fermi level is crossed by the $s$ band at around $k_{\|}=0.8 \AA^{-1}$. For $1 \mathrm{ML}$ and $2 \mathrm{ML} \mathrm{Ag} \mathrm{films} \mathrm{on} \mathrm{V(100),}$ Figs. 3 and 4, states largely localized at the surface (thick lines) also appear in this range of $k_{\|}$. Strong hybridization with vanadium states makes the whole picture rather complicated, and a quantitative interpretation of the STM experiments is not possible at present.

\section{CONCLUSIONS}

Our calculations of the electronic states in few monolayers thick silver films on $\mathrm{V}(100)$ substrate have shown that there is a strong localization of silver-derived states within the Ag layer, due to the mismatch of the electronic structure of the two metals. The localization is particularly noticeable at the $\bar{\Gamma}$ point of the surface Brillouin zone, where quantumwell states of both $s$ character (close to the Fermi level) and $d$ character (at more than $3 \mathrm{eV}$ binding energy) appear on $\mathrm{Ag}$ films. States of $d_{z z}$ symmetry, on the other hand, readily hybridize with vanadium $s$ electrons, which are symmetry compatible. Hybridization effects also become more important away from the center of the Brillouin zone. We have obtained good agreement with photoemission experimental spectra, both in the direction normal to the surface and with the available measurements in off-normal directions.

\section{ACKNOWLEDGMENTS}

This work was supported by the Ministry of Science and Technology of the Republic of Croatia under Contract No. 0098001. We acknowledge useful discussions with M. Milun, P. Pervan, and M. Kralj, and their kind permission to reproduce unpublished experimental spectra.

\footnotetext{
*Email address: radovan@thphys.irb.hr

${ }^{1}$ M. Milun, P. Pervan, and D. P. Woodruff, Rep. Prog. Phys. 65, 99 (2002).

${ }^{2}$ P. D. Johnson, K. Garrison, Q. Dong, N. V. Smith, Dongqi Li, J. Mattson, J. Pearson, and S. D. Bader, Phys. Rev. B 50, 8954 (1994).
}

${ }^{3}$ I. Matsuda, T. Ohta, and H. W. Yeom, Phys. Rev. B 65, 085327 (2002).

${ }^{4}$ J. J. Paggel, T. Miller, and T.-C. Chiang, Science 283, 1709 (1999).

${ }^{5}$ P. T. Sprunger, L. Petersen, E. W. Plummer, E. Lægsgaard, and F. Besenbacher, Science 275, 1764 (1997) 
${ }^{6}$ M. Kralj, P. Pervan, M. Milun, P. Lazić, Ž. Crljen, R. Brako, J. Schneider, A. Rosenhahn, and K. Wandelt, Phys. Rev. B 68, 195402 (2003).

${ }^{7}$ T. Valla, P. Pervan, M. Milun, A. B. Hayden, and D. P. Woodruff, Phys. Rev. B 54, 11786 (1996).

${ }^{8}$ M. Kralj, P. Pervan, M. Milun, T. Valla, P. D. Johnson, and D. P. Woodruff, Phys. Rev. B 68, 245413 (2003).

${ }^{9}$ M. Kralj, A. Šiber, P. Pervan, M. Milun, T. Valla, P. D. Johnson, and D. P. Woodruff, Phys. Rev. B 64, 085411 (2001).

${ }^{10}$ See, for example, S.-Å. Lindgren and L. Walldén, Phys. Rev. Lett. 59, 3003 (1987).

${ }^{11}$ N. V. Smith, N. B. Brookes, Y. Chang, and P. D. Johnson, Phys. Rev. B 49, 332 (1994).

${ }^{12}$ A. Ernst, J. Henk, M. Lüders, Z. Szotek, and W. M. Temmerman, Phys. Rev. B 66, 165435 (2002).
${ }^{13}$ J. M. Carlsson and B. Hellsing, Phys. Rev. B 61, 13973 (2000).

${ }^{14}$ C. M. Wei and M. Y. Chou, Phys. Rev. B 68, 125406 (2003).

${ }^{15}$ M. Kralj, Ph.D. thesis, University of Zagreb, 2003.

${ }^{16}$ M. Kralj (unpublished).

${ }^{17}$ M. Kralj, M. Milun, and P. Pervan, Surf. Sci. 557, 208 (2004).

${ }^{18}$ S. Hüfner, Photoelectron Spectroscopy: Principles and Applications, Springer Series in Solid-State Sciences Vol. 82 (SpringerVerlag, Berlin, 1995).

${ }^{19}$ M. Milun, P. Pervan, B. Gumhalter, and D. P. Woodruff, Phys. Rev. B 59, 5170 (1999).

${ }^{20}$ M. Milun, M. Kralj, and P. Pervan (unpublished).

${ }^{21}$ G. Fuster, J. M. Tyler, N. E. Brener, J. Callaway, and D. Bagayoko, Phys. Rev. B 42, 7322 (2003).

${ }^{22}$ Y. Hasegawa and Ph. Avouris, Phys. Rev. Lett. 71, 1071 (1993). 